\title{
Performance Evaluation of Carrier Aggregation in LTE-A Pro Mobile Systems
}

\author{
Aneta Kolackova ${ }^{1}$, Salwa Saafi ${ }^{1,2}$, Pavel Masek ${ }^{1}$, Jiri Hosek ${ }^{1}$, and Jan Jerabek ${ }^{1}$ \\ ${ }^{1}$ Department of Telecommunications, Brno University of Technology, Brno, Czech Republic \\ ${ }^{2}$ Unit of Electrical Engineering, Tampere University, Finland \\ Email: masekpavel@vutbr.cz
}

\begin{abstract}
Carrier Aggregation (CA) was introduced by the 3GPP, in its Release 10 i.e., Long Term Evolution - Advanced (LTE-A), to address the peak data rate requirement set by the IMT-Advanced standard. As it enables for quick adoption of the fragmented radio spectrum, it was recognized by the telecommunication operators as a game-changing technology for achieving significantly increased data rates. In this paper, we detail how the implementation of $\mathrm{CA}$ with up to five Components Carriers (CCs) impacts the achievable throughput of connected end-users. In the simulation tool Network Simulator 3 (NS-3), the intra-band contiguous CA was implemented for both downlink and uplink channels. In addition, a uniform 2D grid of values that represent the Signal-to-Noise Ratio (SINR) in the downlink with respect to the eNodeB (eNB) i.e., Radio Environment Map (REM) was implemented. As the previously published results for the CA contain mostly the data for the downlink channel, the implemented scenario provides new insights related to the uplink channel communication. Also, in the performance evaluation, we illustrate the expected data rates for the 5G New Radio (NR) systems and compare them with the achieved results in the case of $4 \mathrm{G}$ CA setup.
\end{abstract}

Index Terms-Carrier Aggregation, Cellular Systems, LTEAdvanced, Network Simulator 3, Performance Evaluation.

\section{INTRODUCTION}

Over the course of recent years, the radio spectrum has reached the point, where it is treated as a critical resource for a wide variety of directions going from economic, social to cultural and research. Nonetheless, the principles of how to manage the frequency resources have remained unchanged since the 1980s, mostly due to the complexity of the radio management and immature radio technologies [1]. To overcome the legacy effect of the fragmentation in frequency spectrum, there is a compelling demand for novel techniques allowing for efficient utilization of already available but underused spectrum. The commitment for this change has become recently highly urgent across all the telecommunication companies as the requirements on the radio spectrum are continuously building, mostly due to the enormous increase in wireless traffic [2].

As the amount of transmitted data within mobile networks is expected to exceed the traffic generated by the wired systems soon, today's mobile networks (the fourth generation (4G) networks) can eventually reach the point, where there is no system capacity left, mostly due to the increased capacity per base station (eNodeB, eNB) in Radio Access Network
(RAN) [3]. While standardizing the Long Term Evolution - Advanced (LTE-A) as part of its Release 10, the Third Generation Partnership Project (3GPP) started addressing these requirements by introducing novel techniques for spectral efficiency improvements, network cell density increasing, and exploitation of underutilized radio spectrum resources [2]. One of the LTE-A features for efficient spectrum management is Carrier Aggregation (CA), which consists of simultaneously aggregating multiple frequency fragments, called Component Carriers (CCs). To assure backward compatibility, each of these carriers is configured to be compliant with LTE 3GPP Release 8. As a result, each CC can have a particular bandwidth from those originally supported within the LTE: 1.4, $3,5,10,15$, or $20 \mathrm{MHz}$. By aggregating these CCs, a wider transmission bandwidth will be formed, thus, a higher data rate will be possibly achieved [4].

\section{A. CA From LTE-A to LTE-A Pro}

As mentioned above, CA was first introduced in LTE-A 3GPP Release 10. However, this feature has evolved through the following releases, by adding new CA types or possible configurations. CA types are based on the location of $\mathrm{CCs}$ - they can be either in the same frequency band (i.e., intraband) or in different bands (i.e., inter-band). In case of intraband CA, CCs can be adjacent (i.e., contiguous) or nonadjacent (i.e., non-contiguous). In total, three main CA types are possible: (i) intra-band contiguous, (ii) intra-band noncontiguous, and (iii) inter-band non-contiguous. Concerning CA possible configurations, each band can have a bandwidth class, which is specified by the total number of aggregated Physical Resource Blocks (PRBs) and the maximum number of CCs [5].

In Release 10, only CA inter-band and intra-band contiguous were supported, and the number of CCs was limited to two. Hence, the maximum aggregated bandwidth was set to $40 \mathrm{MHz}$. Intra-band non-contiguous $\mathrm{CA}$ was later introduced in Release 11 [6]. The third CA bandwidth class was further added in Release 12, where User Equipment (UE) can support up to three CCs, thus, a maximum aggregated bandwidth of $60 \mathrm{MHz}$. Releases 13-15 have brought major enhancements in mobile broadband radio performance on top of LTE-A. These enhancements were part of the LTE-A Pro standard, also referred to as $4.9 \mathrm{G}$. Regarding the CA feature additions, the 
number of supported CCs was increased to four in Releases 13 and 14, and then it reached five in Release 15, offering LTE-A Pro networks a maximum aggregated bandwidth of $100 \mathrm{MHz}$ [7]. It became possible also to aggregate licensed and unlicensed frequencies in the downlink, known as Licensed Assisted Access (LAA), and in the uplink, known as enhanced LAA (eLAA) [8].

As it provides improved radio capabilities and allows the use of $100 \mathrm{MHz}$ bandwidth that mobile network operators count to deploy within $5 \mathrm{G}$ sub-6 $\mathrm{GHz}$ networks, LTE-A Pro is considered as able to fulfill, in theory, most of the $5 \mathrm{G}$ sub$6 \mathrm{GHz}$ targets including data rate, latency, and mobility. It also enables LTE networks to offer new sets of services, including IoT applications, vehicular communications for Intelligent transport systems (ITS), and public safety. Based on that, the CA is considered as a key technology enabling some of LTEA Pro technologies and for providing higher quality for LTE networks. This is a promising starting point for future cellular deployments since the first implementations of $5 \mathrm{G}$ systems rely on dual connectivity i.e., data rates from $5 \mathrm{G}$ and LTE radios are combined [9].

\section{B. Available Simulation Tools Enabling CA}

In light of the above, it appears that deploying advanced radio spectrum management techniques becomes unavoidable for telecommunication operators to fulfill the increasing demands of their customers. However, the implementation, verification, and long-term evaluation of newly added features, such as CA, on the existing networks might be expensive, time demanding, and sometimes hazardous. As they can offer highly valuable preliminary results, advanced simulation tools can be used to test and validate new features before deploying them in real mobile systems. More precisely, using a simulation tool to fully control the CA configuration could help the operators not only save time and expenses, but also anticipate possible enhancements. This could be beneficial especially in the case of future $5 \mathrm{G}$ deployments.

The main open source simulation tools implementing both LTE access Evolved Universal Terrestrial Radio Access Network (E-UTRAN) and Evolved Packet Core (EPC) networks, and providing CA functionality are: (i) LTE-Sim and (ii) Network Simulator 3 (NS-3). In LTE-Sim, the network is composed of three kinds of nodes: (i) (UE), (ii) eNB, and (iii) Mobility Management Entity / Gateway (MME/ GW). Additionally, LTE-Sim provides models for the physical (PHY) layer, including Adaptive Modulation and Coding (AMC) scheme, Channel Quality Indicator (CQI) feedback, frequency reuse techniques, and scheduling strategies. At the application layer, four different traffic generators are implemented as well as the management of data radio bearers is supported [10]. Nevertheless, LTE-Sim does not offer to use a TCP model, which is considered as the main drawback of this tool since it shrinks the possible simulation scenarios. Component carriers cannot operate in license-exempt frequency bands, thus, LAA and eLAA functionalities are not supported by LTE-Sim [11].
Next in order, NS-3, more precisely the LTE/EPC Network simulator (LENA) module, provides an implementation of both (i) UE, and (ii) eNB devices, including all the LTE protocol stack layers. On the top of the already supported features by LTE-Sim, NS-3 does provide important LTE design techniques, such as channel modeling with E-UTRAN propagation loss, Radio Resource Management (RRM) algorithms, Medium Access Control (MAC) queues, TCP model, mobility management, Heterogeneous Networks (HetNets), and multiRAT solutions [12].

The extension of NS-3 LENA module supporting the CA feature has been released as part of the official version NS3.27 , offering three different options of configuration based on the degree of CA-related parameters control. In this work, we consider the NS-3.30.1 version as a simulator containing all the CA functionalities needed for the comprehensive evaluation of this feature. Using the latest version of NS-3, it is possible to cover the scenarios, where LTE-A, LTE-A Pro, and preliminary implementation of $5 \mathrm{G}$ New Radio (NR) are deployed.

\section{Our Contribution}

In this article, we describe the available CA extension of NS-3.30.1 LENA module, with the focus on the changes influencing both Control Plane (CP) and Data Plane (DP) implementation for eNB and UE entities. We do also provide a description of the created simulation scenarios and the validation of whether the intended CA configurations do provide a performance boost in comparison with the legacy LTE system without the CA implemented. Since the majority of previous CA implementations focused only on the downlink performance, uplink results will be discussed in this paper as well. The focus is specifically given to two scenarios: (i) the throughput per UE in the case of dynamically changing number of connected UEs for static CC configuration; (ii) the throughput per UE in the case of a static number of UEs with an increasing distance between UEs and the eNB. At the same time, a uniform 2D grid of values that represent the Signal-toNoise Ratio (SINR) in the downlink with respect to the eNB, i.e., Radio Environment Map (REM) is provided to show the TX levels for different communication distances between the eNB and end-users.

\section{LTE-EPC MOdULE AND CA IN NS-3}

This section discusses the key details related to the required modifications applied on LENA module towards the CA functionality in NS-3. Based on this knowledge, we were able to adjust the test suite for our simulation purposes in Section III-A.

\section{A. Requirements Given by $3 G P P$}

To implement the CA technology following the 3GPP recommendations, it is necessary to describe the potential impact on the LTE stack. Based on the proposed functionality, the CA effects mainly the MAC and the radio resource control (RRC). As the backward compatibility between individual 
releases, i.e., between Rel. 8 , Rel. 9 and Rel. 10, needs to be kept, the CC is implemented as a Rel. 8 carrier. Nevertheless, some modifications are requested: (i) extension of the RR messages to process the Secondary Component Carriers (SCC) information, and (ii) MAC to enable simultaneous scheduling on a number of CCs. To enable the backward compatibility across 3GPP released in LTE-A or LTE-A Pro, each UE establishes the cell connection based on Rel. 8 procedure, i.e., a cell search and selection, followed by the system information acquisition phase and the random access procedure [13].

\section{B. LENA Module Changes Towards CA Functionality}

As it was mentioned in the opening section, the CA technology allows telecommunication operators to aggregate radio resources from different carriers through available bandwidth to achieve higher network throughput. Each CC can have different available bandwidth, even in the LENA module. With CA technology, more serving cells are used at the same time to transmit the data. The coverage of utilized cells (eNBs) varies as each $\mathrm{CC}$ can use different frequency bands that have different path loss coefficient. The RRC connection is always established by one cell, called "primary serving cell". If the Primary Component Carrier (PCC) does not have enough capacity to serve all the traffic, the secondary carrier can offload some traffic, while the RRC connection is still using the primary carrier. The CA technology has impact on different layers of the LTE protocol stack. The biggest impact is on the MAC layer as the scheduler located there must be able to handle different number of CCs. Another impact can be found on the RRC layer, where new messages related to the secondary carrier configuration and measurements reporting are defined. In contrast, there is almost no impact on the PHY layer and Packet Data Convergence Protocol layers (PDCP) and a minor impact on the RLC layer in the form of an enlarged buffer [14].

By default, the CA feature is disabled in the NS-3 LENA module. Therefore, to activate it, the Boolean attribute ns3::LteHelper: :UseCa needs to be set to true. There are three ways how CA simulation can be specified: (i) delegating the configuration to LteHelper, (ii) using CcHelper, or (iii) creating a sta: :map of ComponentCarrier objects. For our purposes, we used the third option, that allows us full level of control over CCs configuration (to set uplink bandwidth, downlink bandwidth, uplink earfen, downlink earfen). The CCs attributes are handled by the delegated classes ComponentCarrierEnb and ComponentCarrierUe, such as the ones concerning a specific carrier, pointers to MAC, PHY, a scheduler or fractional frequency reuse algorithm instances, which were previously included in LteEnbNetDevice or LteUeNetDevice. Other physical parameters of each CC are provided by the class ComponentCarrier. To be able to control CCs operations, the Component Carrier Manager (CCM) is included on both the eNB and UE [15].

Changes regarding the $\mathrm{CA}$ functionality can be divided into $\mathrm{CP}$ and DP. CP has the same implementation for eNB and UE.
For each CC, the PHY and the MAC are directly associated to RRC instance. In its turn, the RRC instance is connected to the CCM (LteUe/EnbComponentCarrierManager), which divides the traffic distribution between CCs. The number of CCs is fixed when the simulation starts, but it is only the PCC that is working, the other CCs are in use after UE is in connected state.

DP differs from eNB and UE point of view. LteEnbComponentCarrierManager provides main functions even for UEs, this manager processes the Buffer Status Report (BSR), which carries the information on how much data is in UE buffer to be sent out. Each time a Radio Link Control (RLC) instance sends a BSR, LteEnbComponentCarrierManager receives it and sends it to the MAC instance. In the case of UE, each time a BSR is generated, LteUeComponentCarrierManager uses PCC to send it to eNB. After receiving a BSR from UE, eNB forwards it to LteEnbComponentCarrierManager, which uses an implemented algorithm to process it. As a result, some of the traffic is split between different CCs based on the BSR and assuming that the primary carrier does not have sufficient capacity [15].

\section{REFERENCE SCENARIO IN NS-3}

This section provides a discussion on the communication scenario, where the evaluation of the carrier aggregation features is performed. The obtained data is processed and discussed in terms of the network throughput, communication distance, and signal quantity of this particular wireless system.

\section{A. Simulation topology and parameters}

The topology used for testing purposes is based on the test suite lte-carrier-aggregation. This test suite can be used as a separate program or in combination with LENA examples, i.e., lena-simple or lena-simple-epc. We modified this test suite to be able to set the parameters which would enable us to reach the maximal output with CA in NS3. Firstly, we focused on the number of CCs. We fixed it at five, which is the maximum number of CCs in LTE-A Pro systems. Our modifications allowed us to choose uplink and downlink bandwidth as well as uplink and downlink earfen. In the simulation, we focused on intra-band contiguous $\mathrm{CA}$ only. Therefore, we selected for this scenario, the frequency band 40, i.e., $2300 \mathrm{MHz}$. This whole modification will be built on/with the LENA module, see Fig. 1. All simulations are performed on the Uu connection, which is the air interface connecting the eNodeB with UEs.

The CA, as defined by 3GPP, can be used with Time Division Duplex (TDD) and also Frequency Division Duplex (FDD). There can be up to $5 \mathrm{CCs}$ and each of them can be allocated with the bandwidth of $1.4,3,5$, 10,15 , or $20 \mathrm{MHz}$, with maximum limit of $100 \mathrm{MHz}$ for all CCs. Further, the system bandwidth is assigned as follows: (i) $1.4 \mathrm{MHz}(6 \mathrm{RBs})$, (ii) $3 \mathrm{MHz}(15 \mathrm{RBs})$, (iii) $5 \mathrm{MHz}$ (25 RBs), (iv) $10 \mathrm{MHz}$ (50 RBs), (v) $15 \mathrm{MHz}$ (75 RBs), and 


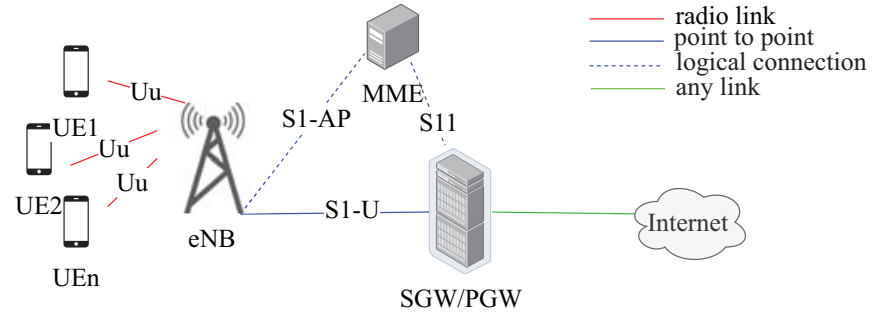

Fig. 1. LTE-EPC simulation model LENA NS-3. For the purpose of this paper, one eNB and 20 UEs with the same RB specification are implemented

(vi) $20 \mathrm{MHz}(100 \mathrm{RBs})$ [5]. In the simulation scenario, the bandwidth was set according to the number of RBs - in one $\mathrm{CC}$, the maximum number of RBs is 100 . Other parameters, which were set in our carrier aggregation scenario apart from the bandwidth and CCs are: (i) the distance, (ii) the number of UEs, and (iii) the frequency. The frequency is always set for the particular simulation so CCs operate within the same frequency band, so called intra-band contiguous. The remaining parameters are listed in the Tab. I and discussed through the following lines, where the scenarios considered to achieve maximum performance are explained.

\section{B. Simulation Scenarios}

The first scenario is focused on getting maximal possible downlink/uplink output for a CA - enabled 4G system simulated in NS-3 to be able to compare it with $5 \mathrm{G}$ prediction for 2023. We chose for this scenario frequency $2300 \mathrm{MHz}$ of band 40 , which is $100 \mathrm{MHz}$ wide and because of this we could dedicated each of CCs $20 \mathrm{MHz}$ bandwidth. To achieve maximal output we used $5 \mathrm{CCs}$ and measured it with the minimal real distance between eNodeB and UE, i.e., $50 \mathrm{~m}$. The number of UEs was set in the range from 1 to 20 , with the increasing number of UEs, the time length of the simulation also increases, so due to the time-consuming nature of the measurements, the number was set up to 20 UEs maximum. The Tab. I shows the other selected parameters containing the complete list of the distances between the eNB and UEs. The simulation results are further presented in the following section.

The second scenario was targeted to get dependence of the communication distance between the UEs and the eNodeB when using CA. The measurement was performed for both downlink and uplink. For downlink, we used 5 CCs with $20 \mathrm{MHz}$ bandwidth and for uplink $3 \mathrm{CCs}$ also with $20 \mathrm{MHz}$ bandwidth, based on the recommendations of 3GPP TS 36.101 V16.4.0 for band 40 [7]. The distance between the eNB and UEs was defined in the following steps: (i) $50 \mathrm{~m}$, (ii) $250 \mathrm{~m}$, (iii) $500 \mathrm{~m}$, (iv) $750 \mathrm{~m}$, (v) $950 \mathrm{~m}$, (vi) $1050 \mathrm{~m}$, (vii) $1250 \mathrm{~m}$, (viii) $1450 \mathrm{~m}$, (ix) $1450 \mathrm{~m}$, (x) $1650 \mathrm{~m}$, (xi) $1850 \mathrm{~m}$, and (xii) $2050 \mathrm{~m}$. The other parameters match the data in the Tab. I.

As a third output of the performed simulations, with same parameters as it was for first and second scenarios, is REM. REM is a uniform 2D grid of values that represent the Signalto-noise Ratio (SINR) in downlink with respect to the eNB
TABLE I

SIMULATION PARAMETERS OF INTENDED SCENARIOS.

\begin{tabular}{|l|c|}
\hline \hline & Scenarios Configuration \\
\hline \hline Bandwidth & $20 \mathrm{MHz}$ \\
\hline Number of RB & 100 \\
\hline Number of UEs & 20 \\
\hline Number of CCs & $5 \mathrm{DL} / 3 \mathrm{UL}$ \\
\hline Frequency & $2300 \mathrm{MHz}$ \\
\hline Band n. & 40 \\
\hline eNodeB TX Power & $30 \mathrm{dBm}$ \\
\hline Noise Figure - eNB & $5 \mathrm{dBm}$ \\
\hline UEs TX Power & $23 \mathrm{dBm}$ \\
\hline Noise Figure - UE & $9 \mathrm{dBm}$ \\
\hline MCS & $64 \mathrm{QAM}$ \\
\hline Mobility Model & Constant (i.e., no mobility) \\
\hline $\begin{array}{l}\text { Distance in meters } \\
\text { from eNodeB }\end{array}$ & $\begin{array}{c}50,250,500,750,950,1050,1250, \\
1450,1650,1850,2050 \mathrm{~m}\end{array}$ \\
\hline Scheduler Type & PfFfMacScheduler \\
\hline
\end{tabular}

that has the strongest signal at each point. The given values have been processed into a REM shown in Fig. 6 .

\section{Simulation Results}

By using our modified carrier aggregation test suite, we obtained data which was further processed using Matlab and plotted into the graphs, see Figs. 2-5. In addition, a REM plot was created to better illustrate the obtained simulation results with respect to the SINR, see Fig. 6.

The Fig. 2 discusses downlink performance (throughput in Mbps) per UE. The scenario in question uses $5 \mathrm{CCs}$ and shows the performance of legacy LTE with the PCC only, i.e., without the CA - this is also described as no Supplemental Downlink (SDL) approach. The remaining data represents the LTE scenarios with one, two, three, and four SDL carriers. This setup provides us the maximal downlink performance for LTE using CA. The maximal throughput for $1 \mathrm{UE}$ with $5 \mathrm{CCs}$, bandwidth $20 \mathrm{MHz}$, and distance $50 \mathrm{~m}$ was $376 \mathrm{Mbps}$. Because of uniform distribution, which is used in simulations, the cell's capacity is uniformly distributed between the competing UEs, so the per terminal throughput will be $376 / 10$ in case of 10 UEs and decreases to $376 / 20$ in case of 20 competing UEs. Overall, the network throughput is increasing linearly based on the number of used CCs. Based on [16], the average speed provided by $5 \mathrm{G}$ networks in 2023 should reach $575 \mathrm{Mbps}$ and so should be 13 times higher than we are experiencing in current cellular systems on daily basis in real world. This is for the scenarios where the frequencies bellow $6 \mathrm{GHz}$ with bandwidth $100 \mathrm{MHz}$ in case of the non-standalone (NSA) are selected. This prediction is also shown in Fig. 2 for a better comparison with respect to the simulated scenarios.

In our study, we also focused on the uplink performance (throughput in Mbps) per UE. This scenario is particularly important as there is a lack of performance in comparison with $5 \mathrm{G}$ if we consider the currently available research works. With many industries having massive data that needs to be uploaded for back-end processing such as $4 \mathrm{~K}$ HD live stream 


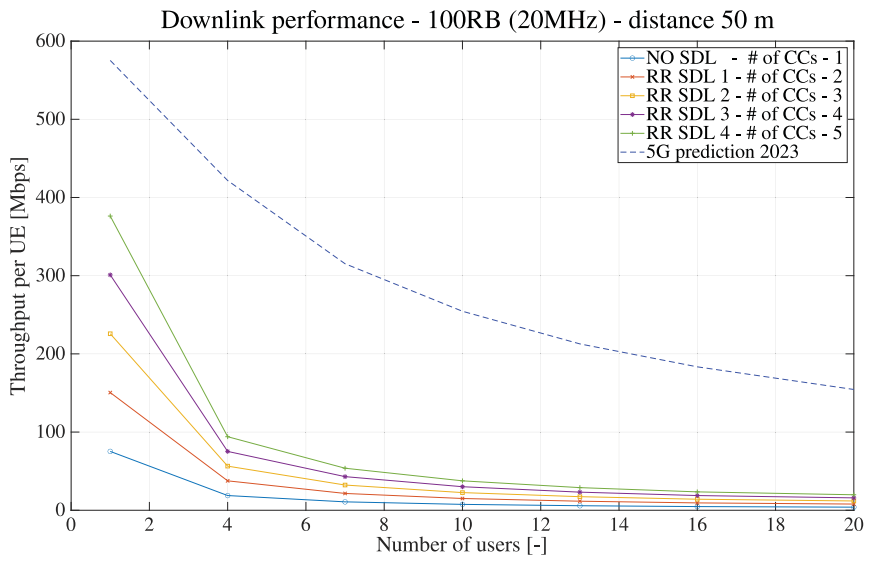

Fig. 2. Downlink performance $100 \mathrm{RB}$ - bandwidth $20 \mathrm{MHz}$ with distance $50 \mathrm{~m}$ from eNodeB.

or unmanned mining vehicles, this scenario shows us that the LTE with CA is insufficient. The expectations for $5 \mathrm{G}$ are for uplink similar as for downlink - 13 times higher. The results shown in the Fig. 3 represent the maximal possible configuration for LTE CA uplink in NS-3. The maximal throughput for $1 \mathrm{UE}$ with $3 \mathrm{CCs}$, bandwidth $20 \mathrm{MHz}$, and distance $50 \mathrm{~m}$ was $225 \mathrm{Mbps}$.

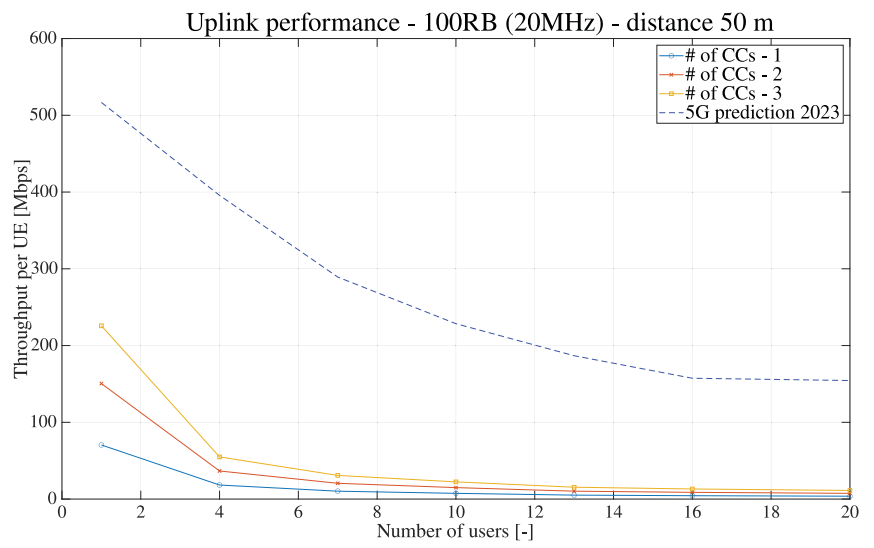

Fig. 3. Uplink performance $100 \mathrm{RB}$ - bandwidth $20 \mathrm{MHz}$ with distance $50 \mathrm{~m}$ from eNodeB

The second scenario does focus on dependence of the distance of UEs to the eNB for both downlink and uplink. Tab. I shows the implemented distance steps and TX power of each UEs and eNB. For all the UEs, we configured the same TX power, i.e., $23 \mathrm{dBm}$. The configuration of the TX power in case of the eNB was set to $30 \mathrm{dBm}$.

The downlink performance is shown in Fig. 4. The most noticeable outcome is a difference between $1 \mathrm{UE}$ and more UEs due to uniform distribution. $1 \mathrm{UE}$ could reach throughput $376 \mathrm{Mbps}$ at measured distances from $50 \mathrm{~m}$ up to $1250 \mathrm{~m}$, and then the throughput is linearly decreasing in distance above $1250 \mathrm{~m}$ and up to $1650 \mathrm{~m}$. Same throughput as for $1650 \mathrm{~m}$ was measured for $1850 \mathrm{~m}$ and then at the distance $2050 \mathrm{~m}$ the throughput dropped even more to $234 \mathrm{Mbps}$.

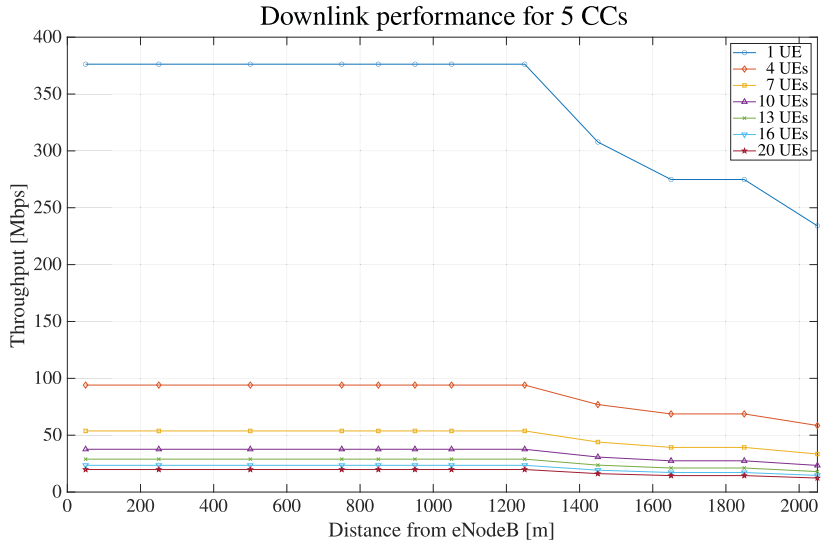

Fig. 4. Downlink performance $100 \mathrm{RB}$ - bandwidth $20 \mathrm{MHz}$ based on distance from eNodeB and number of UEs.

The uplink is shown in the Fig. 5. The distance dependence is observable from smaller distances. Because of the uniform distribution, a significant drop is noticeable based on number of connected UEs. For $1 \mathrm{UE}$ we obtained throughput $225 \mathrm{Mbps}$ up to $500 \mathrm{~m}$ from UE to eNB. However, with increasing distance the overall throughput decreases. The reduction in data throughput begins to occur after exceeding a distance of $500 \mathrm{~m}$. The throughput degradation is due to the free-path loss, which increases with the distance. In case of these simulations, the TX power was maintained fixed, as Tab. I shows, so the Signal to Noise Ration (SNR) at the receiver side varies with the changing distance between the TX and RX. In general, if the SNR is high enough, the link adaptation selects Modulation Coding Scheme (MCS) with high modulation orders and high coding rates, which results in transmissions with high spectral efficiency, while at lower SNRs, the link adaptation has to select modulations with smaller orders and more Forward Error Correction (FEC) redundancy bits which leads to lower spectral efficiency.

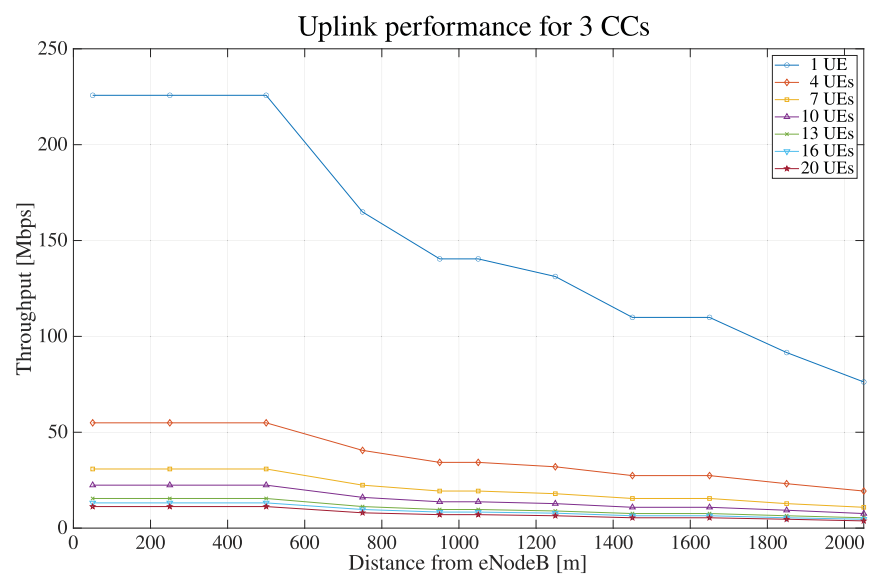

Fig. 5. Uplink performance $100 \mathrm{RB}$ - bandwidth $20 \mathrm{MHz}$ based on distance from eNodeB and number of UEs.

As it was mention above, simulation parameters used for both scenarios were processed for obtaining 
results recorded in the ACSII file. By using class RadioEnvironmentMapHelper, it was possible to create output in the form of REM. The RadioEnvironmentMapHelper uses the bandwidth parameters and the frequency of the band used to determine the spectrum model. The Fig. 6 shows the REM for 5 CCs with a bandwidth of $20 \mathrm{MHz}(100 \mathrm{RBs})$ in band 40 . The obtained REM confirmes the reason why the distance influenced the throughput at a distance above $1250 \mathrm{~m}$ in downlink. The SINR value from this distance has dropped to a level that does not guarantee a reliable transmission.

Any other transmitters causing interference were not considered, because the whole simulation was in an unobstructed environment.

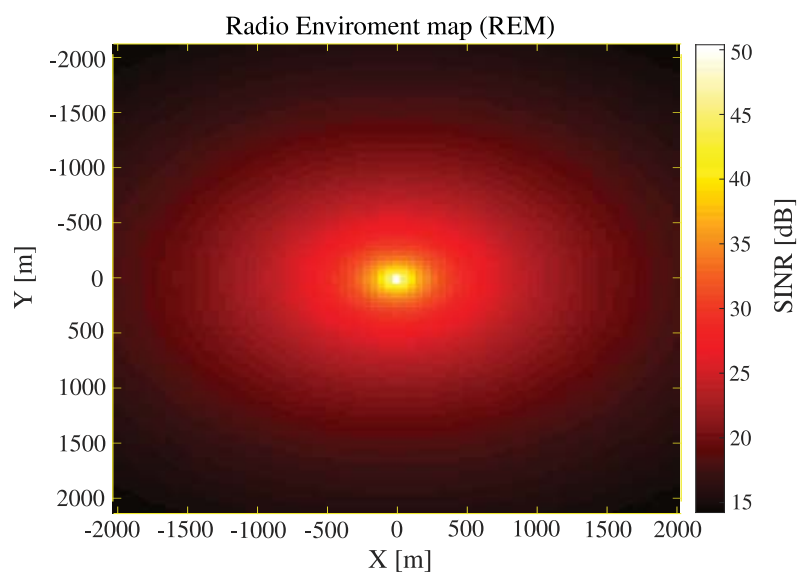

Fig. 6. REM obtained for scenario with bandwidth $20 \mathrm{MHz}(100 \mathrm{RB})$.

\section{CONCLUSiOnS}

The reported outputs in this paper represent the simulation results obtained using the LTE/EPC module in NS-3. As the utilized module does support the CA functionality, we have implemented the simulation scenarios with $\mathrm{CA}$, where up to five CCs were used for both downlink and uplink communication channels. More precisely, in our scenarios, we have utilized the intra-band contiguous $\mathrm{CA}$ approach as the frequency band 40 was selected and implemented following the 3GPP TS 36.101 V16.4.0, i.e., for downlink 5 CCs with $20 \mathrm{MHz}$ bandwidth and for uplink $3 \mathrm{CCs}$ with the same maximum bandwidth were used.

As the other recent research works dealing with the CA are mostly focused on the downlink channel, the implemented scenarios provide new insights related to the uplink communication modelled in the latest version of the NS-3, i.e., NS3.30.1. The results confirm, for the selected frequency band 40 , the expectations as the achieved data rates go up to $220 \mathrm{Mbps}$ for the uplink and $380 \mathrm{Mbps}$ in case of the downlink. While comparing the obtained results for the legacy LTE and LTEA Pro technologies, we have also added the expected data rates for the $5 \mathrm{G}$ NR systems (following the most recent white papers and studies from the key telco players) to understand the performance gaps, see Figs. 2-5.

On top of the mentioned results, we have also provided a uniform 2D grid of values that represent the SNR in the downlink channel. This is to show the expected TX power levels for the end-devices mostly in case of the simulation scenarios, where the distance between the eNB and UEs is more than $1.2 \mathrm{~km}$ as we do consider the standard TX power for both the eNB and UEs i.e., $30 \mathrm{dBm}$ and $23 \mathrm{dBm}$, respectively and band 40 with frequency $2300 \mathrm{MHz}$.

\section{ACKNOWLEDGMENT}

The authors gratefully acknowledge funding from the European Union's Horizon 2020 Research under the Marie Sklodowska Curie grant agreement No.813278 (A-WEAR: A network for dynamic wearable applications with privacy constraints, http://www.a-wear.eu/). This work was also supported by the Technology Agency of Czech Republic project No. TK02030039.

\section{REFERENCES}

[1] M. K. Afzal, Y. B. Zikria, S. Mumtaz, A. Rayes, A. Al-Dulaimi, and M. Guizani, "Unlocking 5G Spectrum Potential for Intelligent IoT: Opportunities, Challenges, and Solutions," IEEE Communications Magazine, vol. 56, no. 10, pp. 92-93, 2018.

[2] K. Chatzikokolakis, P. Spapis, A. Kaloxylos, and N. Alonistioti, "Toward Spectrum Sharing: Opportunities and Technical Enablers," IEEE Communications Magazine, vol. 53, no. 7, pp. 26-33, 2015.

[3] Cisco, VNI, "Cisco Visual Networking Index: Forecast and Trends, 2017-2022 White Paper,” White Paper, 2019.

[4] G. Yuan, X. Zhang, W. Wang, and Y. Yang, "Carrier Aggregation for LTE-Advanced Mobile Communication Systems," IEEE communications Magazine, vol. 48, no. 2, pp. 88-93, 2010.

[5] G. d. 1. Roche, A. Alayn-Glazunov, and B. Allen, LTE-Advanced and Next Generation Wireless Networks: Channel Modelling and Propagation, 1st ed. Wiley Publishing, 2012.

[6] 3GPP, "Evolved Universal Terrestrial Radio Access (E-UTRA); Carrier aggregation enhancements; User Equipment (UE) and Base Station (BS) radio transmission and reception, TR 36.823 V11.0.1," 2013.

[7] — - "User equipment (ue) radio transmission and reception (release 10), 3gpp ts 36.101," V16, vol. 4, 2019.

[8] Y. Gao, Q. Huang, S. Xu, H. Li, Z. Li, and W. Tang, "Experimental Performance Evaluation and Analysis of LAA and Wi-Fi Coexistence in the Unlicensed Spectrum," in 2016 IEEE Globecom Workshops (GC Wkshps). IEEE, 2016, pp. 1-6.

[9] H. Holma, A. Toskala, and T. Nakamura, 5G Technology: 3GPP New Radio. John Wiley \& Sons, 2020.

[10] G. Piro, L. A. Grieco, G. Boggia, F. Capozzi, and P. Camarda, "Simulating LTE Cellular Systems: An Open-Source Framework," IEEE Transactions on Vehicular Technology, vol. 60, no. 2, pp. 498-513, Feb 2011.

[11] F. Capozzi, G. Piro, L. A. Grieco, G. Boggia, and P. Camarda, "Downlink Packet Scheduling in LTE Cellular Networks: Key Design Issues and a Survey," IEEE Communications Surveys \& Tutorials, vol. 15, no. 2 pp. 678-700, 2012.

[12] G. Piro, N. Baldo, and M. Miozzo, "An LTE Module for the NS3 Network Simulator," in Proceedings of the 4th International ICST Conference on Simulation Tools and Techniques. ICST (Institute for Computer Sciences, Social-Informatics and , 2011, pp. 415-422.

[13] C. Cox, An Introduction to LTE: LTE, LTE-Advanced, SAE and 4G Mobile Communications. John Wiley \& Sons, 2012.

[14] NS-3 Project, "NS-3 Network Simulator Model Library," Release ns3.30.x, 2019.

[15] B. Bojovic, M. D. Abrignani, M. Miozzo, L. Giupponi, and N. Baldo, "Towards LTE-Advanced and LTE-A Pro Network Simulations: Implementing Carrier Aggregation in LTE Module of NS-3," in Proceedings of the Workshop on Ns-3, ser. WNS3 17. New York, NY, USA: Association for Computing Machinery, 2017, p. 6370. [Online]. Available: https://doi.org/10.1145/3067665.3067669

[16] Cisco, VNI, "Cisco Visual Networking Index: Forecast and Trends, 2018-2023," White Paper, vol. 1, 2020. 